\title{
AC 2007-1074: INFLUENTIAL FACTORS AFFECTING THE ATTRACTION AND RETENTION OF MINORITY FACULTY IN ENGINEERING AND TECHNOLOGY PROGRAMS
}

\section{Shonda Bernadin, Georgia Southern University}

Shonda L. Bernadin is an Assistant Professor of Engineering Studies in the Department of Mechanical \& Electrical Engineering Technology at Georgia Southern University. Dr. Bernadin received her B.S. degree in Electrical Engineering from Florida A\&M University in 1997, her M.S. degree in Electrical and Computer Engineering from University of Florida in 1999, and her Ph.D. degree from the Department of Electrical and Computer Engineering at Florida State University in 2003.

\section{Frank Atuahene, Georgia Southern University}

Dr. Frank Atuahene is an Assistant Professor and the Coordinator of the Construction Management Program in the Department of Construction Management and Civil Engineering Technology at Georgia Southern University. Dr. Atuahene has a B.Sc (Hon) degree from the University of Science and Technology in Ghana (1979), a Master of Civil Engineering degree from the University of Delaware (1983), a Master of Engineering degree (Industrial Engineering) from Penn State University (1994) and a Ph.D in Civil and Environmental Engineering from Rutgers University, The State University of New Jersey (1998). 


\title{
Influential Factors Affecting the Attraction and Retention of Minority Faculty in Engineering and Technology Programs
}

\begin{abstract}
This paper highlights research that explores the key factors that affect the attraction and retention of minority faculty in engineering and technology programs in rural communities, in particular southern rural communities. It is well known that the pool of minority applicants, specifically black applicants, for faculty positions in engineering and technology programs is very small. Furthermore, the attraction and retention of qualified minority faculty to teach in engineering and technology programs in rural communities (that is, communities with a population of less than $30,000)$ is even more limited. There are several factors that contribute to this phenomenon, including university classification, department culture, student demographics, and geographical location. We focus on the attraction and retention problem of minority engineering faculty at a comprehensive university in a rural community in southeast Georgia. The target institution has successfully recruited and retained minority faculty members at a steady rate over the last five years. Approximately, $16 \%$ of its total faculty are minorities. These observations initiated the basis of this study. We began to question the nature of this phenomenon and ponder the unique characteristics of this university that fueled these results. Appropriate data was collected, analyzed and used to identify contributing factors that lead to the attraction and retention of minority more specifically, black Engineering and Technology faculty members at this institution. Using several data analysis techniques, we show that there is a high correlation between diversity (in terms of students, faculty and the academic learning environment) and the attraction and retention of black engineering and technology faculty members. These data indicate that the diversity model implemented at this university is successful in attracting and retaining minority faculty members. Consequently as a result of this work, similar faculty diversity models can be developed and used to increase faculty diversity at other academic institutions.
\end{abstract}

\section{Background}

The attraction and retention problem of minority faculty members is an issue at many colleges and universities across the nation. It is obvious that to stay competitive and attract high-quality students many academic institutions must support diversity due to the challenge of producing "global citizens". . However, increasing diversity must also incorporate a diverse faculty population that actively encourages diverse learning environments. Hence, the focus on the issue of adequately addressing the attraction and retention of qualified minority faculty members.

Many universities have adopted successful strategies for attracting minority faculty members including the use of "aggressive individualized recruiting" and attractive salaries and compensation packages ${ }^{2}$. However, some research suggests that once minorities join a faculty, the likelihood of retention decreases due to several factors including a lack of acculturation by the institution ${ }^{3}$. In essence, many minority faculty members feel a sense of value and appreciation during the initial hiring period, but over time they feel disconnected and 
undervalued. Faculty beliefs and attitudes about diversity and diverse learning environments contribute to their attraction to certain university settings ${ }^{4}$. For instance, learning environments that actively support and encourage multicultural activities are more likely to attract and retain diverse student and faculty populations. This, in turn, creates an environment that facilitates learning and produces competitive global thinkers and productive members of the global society.

Furthermore, faculty members are the "face" of the university. They are an integral part of the academic environment and can be a crucial recruiting tool for attracting more diverse students. For Engineering and Technology fields of study there is a strong national push to attract more minorities to these fields. Having a diverse faculty can help institutions of higher education achieve this national goal and recruit top-achieving students who will develop into productive "world-class" citizens.

Georgia Southern University (GSU) was chosen for this study because it has been steadily increasing the number of minority faculty members that it hires in all fields, including Engineering and Technology, as shown in Figure 1, over the past six years, coinciding with university-wide administrative changes, like the hiring of a new president. The university strategically repositioned itself to a higher level of competitiveness by adopting a newly developed academic model for higher learning. This new model supports and encourages diversity through academic and social engagement.

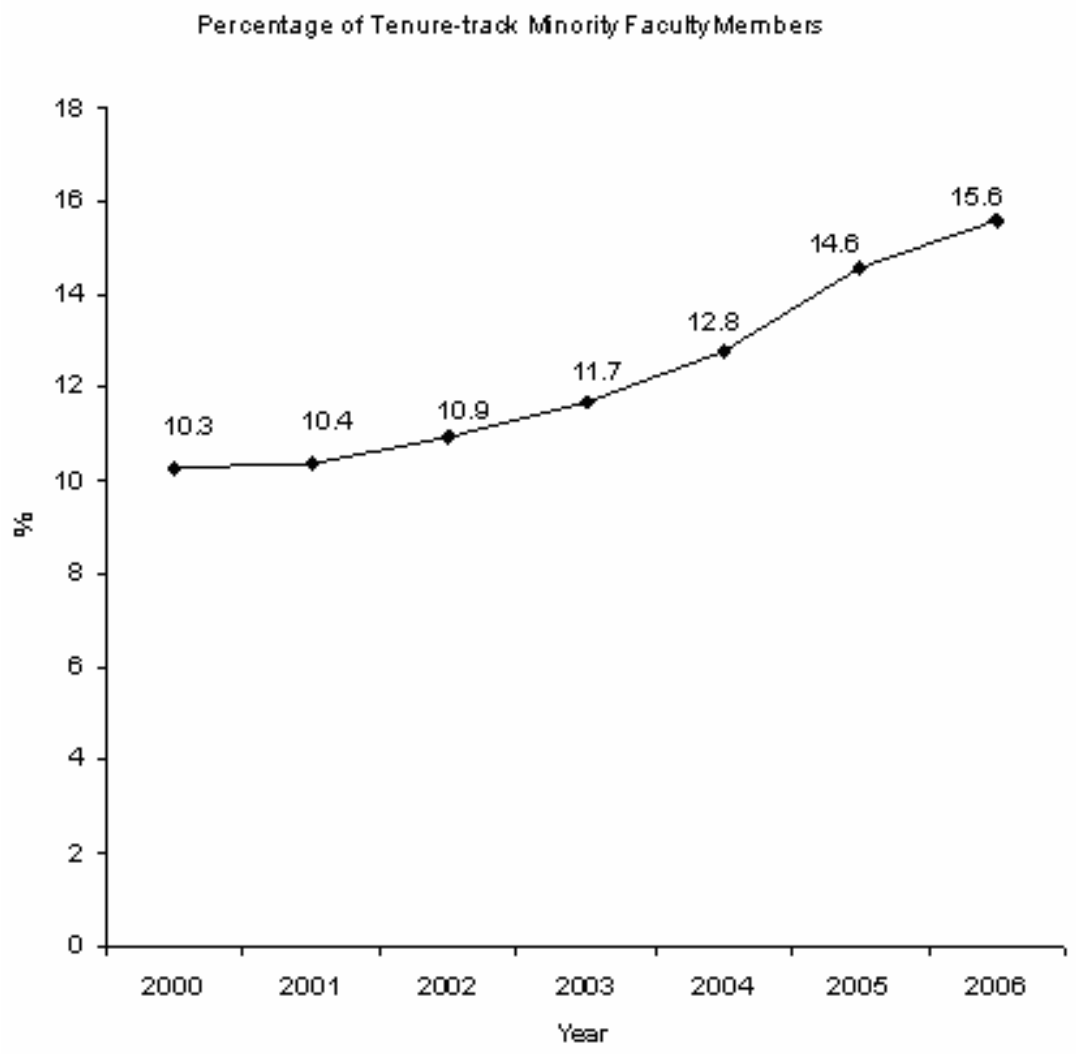

Figure 1-Percentage of Minority Faculty Members since 2000

There are unique features about GSU that attract and retain qualified minority applicants at a relatively successful rate. In this work, some key factors that may contribute to the decision 
making process of minority faculty members to join the faculty are explored by posing the following research questions:

1. How does Engineering and Technology faculty members' likelihood to join the faculty at a comprehensive regional university differ as a function of diversity, department, institution and economic characteristics?

2. How does Engineering and Technology faculty members' likelihood to remain on the faculty at a comprehensive regional university differ as a function of diversity, department, institution and economic characteristics?

3. Do Engineering and Technology faculty members' beliefs about diversity influence their decision to join faculty at this university?

4. Does faculty members' participation in diversity-related events on campus affect their decision to remain a faculty member at this university?

These questions are explored in this study using data collection and analysis techniques. A brief description of the university mission is given in the next section, which provides insight about the academic environment supported by this institution. Methods used for data collection and analyses are described followed by a description of the survey instrument used to collect appropriate data. Data analysis techniques are highlighted followed by a detailed discussion and conclusions.

\section{University Context}

The following statement is a direct quotation from the Georgia Southern University handbook ${ }^{5}$ :

"Georgia Southern University is a public comprehensive university devoted to academic distinction in teaching, scholarship, and service. The University's hallmark is a culture of engagement that bridges theory with practice, extends the learning environment beyond the classroom, and promotes student growth and life success. Its nationally accredited academic programs in the liberal arts, sciences, and professional studies prepare a diverse and select undergraduate and graduate student population for leadership and service as world citizens. Faculty, staff, and students embrace core values expressed through integrity, civility, kindness, collaboration, and a commitment to lifelong learning, wellness, and social responsibility.

Central to the University's mission is the faculty's dedication to excellence in teaching and the development of a fertile learning environment exemplified by a free exchange of ideas, high academic expectations, and individual responsibility for academic achievement. The faculty members at this university are teacher-scholars whose primary responsibility is the creation of learning experiences of the highest quality, informed by scholarly practice, research, and creative activities. 
The University offers a student-centered environment enhanced by technology, transcultural experiences, private and public partnerships, and stewardship of a safe residential campus recognized for its natural beauty."

Clearly, diversity and multicultural experiences are a recurring theme in this mission statement. The facilitation of learning marked by unique cultural learning experiences is also strongly emphasized. In order for this mission to be fulfilled, mechanisms of support must exist that provide direction for effective teaching, learning, and the incorporation of diversity in the classroom. The Center for Excellence in Teaching (CET) and the Multicultural Center are both university entities that actively engage in providing such opportunities for the faculty, staff and students. These are exemplary strategies that are used to increase diversity and enhance the desired learning environment provided by this university.

\section{Data and Methodology}

Most documented research available on faculty diversity at institutions of higher learning is typically tailored for specific institutions. Little work was found that highlighted generalized factors in faculty members' decision to join the faculty at an institution. Through background research, faculty interviews and personal experiences, certain factors that may contribute to the attraction and retention of minority faculty members were identified. A survey was developed based on these factors. The data collection process was based on the one performed by Mayhew and Grunwald ${ }^{4}$.

\section{Survey Instrument}

A survey was created and administered to black faculty members at this institution. Results were collected and analyzed for significant influential factors that contribute to the decision-making process of a black faculty member to teach at this institution. Diversity, Departmental, Institutional, and Economic factors were considered influential in this survey. These categories were selected based on preliminary research collected from Engineering and Technology faculty members. The diversity category measured attitudes about diverse student population, faculty diversity and multicultural campus diversity. The departmental category measured faculty members' attitudes about departmental culture, research opportunities, and course workload. Institutional measures included a faculty member's perception towards factors such as university reputation, mission, vision statements, compensation and benefits. The economic category measured attitudes about economic stability, geographical location, and city demographics as influential factors in their decision-making process. Table 1 lists all of the factors and their corresponding factor numbers.

It was important to obtain a high survey response rate among E\&T black faculty members, particularly since our interests focused on the attraction and retention of minority faculty in Engineering and Technology (E\&T). At this institution, black faculty composed of approximately $10 \%$ of the total E\&T faculty. In this study a 100\% response rate was achieved for black E\&T faculty members, however due to the small size of the department, the total sample size was very limited $(\mathrm{n}=2)$. Consequently, other black faculty members in all academic departments were solicited for survey interviews to increase the total sample size and as a means 
of comparison. A 4-point agreement scale was used to measure the variables (4- Strongly Agree, 3- Somewhat Agree, 2- Somewhat Disagree, 1-Strongly Disagree)

Table 1: Attraction and Retention Factors for Faculty Members

\begin{tabular}{|c|l|}
\hline$\underline{\text { Factor }}$ & Factor \\
\cline { 2 - 2 } & \\
\hline 1 & Diverse student population: \\
\hline 2 & Faculty diversity: \\
\hline 3 & Geographical location: \\
\hline 4 & Department culture: \\
\hline 5 & Multicultural campus environment: \\
\hline 6 & Professional Development Opportunities: \\
\hline 7 & Research Opportunities: \\
\hline 8 & Course Workload: \\
\hline 9 & Size of University: \\
\hline 10 & University Reputation: \\
\hline 11 & Employment Stability: \\
\hline 12 & University Mission and Vision Statements: \\
\hline 13 & Competitive Compensation and Benefits: \\
\hline 14 & Community Support and Service: \\
\hline 15 & Economic Stability and Quality of life: \\
\hline 16 & Demographics/Population of city: \\
\hline 17 & Sustained Employment: \\
\hline
\end{tabular}

\section{Data Analysis Techniques}

For the E\&T department, the sample size was extremely small. Of the 693 total faculty members at this institution, 50 are black and only 2 of these are E\&T faculty members. Surveys were sent to black faculty members and 9 responses were received. To date, the survey response rate was $18 \%$. Due to this low rate, hypothesis testing of the results using a Chi-Square Goodness-of-fit test proved insignificant. However, exploratory data analysis methods and comparison tests were performed on the data collected using significance algorithms.

Based on the defined four categories (diversity, departmental, institutional, economic), a factor was determined to have very high significance if its composite score was over $90 \%$. A factor had high significance if its composite score was over $80 \%$ and average significance if its score is over $75 \%$. Scores less than $75 \%$ yielded low significance.

Other comparisons were made using signal interpretations and analyses of the data. Data was converted to its signal representation and signal analysis techniques were performed to make result comparisons. A polynomial fit algorithm was applied to the data collected to make observations on possible trends. The next section describes the performance results. 


\section{Results}

The significance algorithms were performed on the data collected and the results are highlighted in this section. Table 2 lists the level of significance for the initial attraction of E\&T faculty members for each of the factors in the four categories.

Table 2: Significance of Factors for E\&T Faculty

\begin{tabular}{|c|c|c|c|}
\hline Category & \multicolumn{2}{|c|}{ Factor Factor } & Level of Significance \\
\hline \multirow[t]{3}{*}{ DIVERSITY } & 1 & Diverse student population: & high \\
\hline & 2 & Faculty diversity: & average \\
\hline & 5 & Multicultural campus environment: & high \\
\hline \multirow{3}{*}{ DEPARTMENTAL } & 4 & Department culture: & low \\
\hline & 7 & Research Opportunities: & low \\
\hline & 8 & Course Workload: & low \\
\hline \multirow[t]{6}{*}{ INSTITUTIONAL } & 6 & Professional Development Opportunities: & average \\
\hline & 9 & Size of University: & low \\
\hline & 10 & University Reputation: & low \\
\hline & 11 & Employment Stability: & low \\
\hline & 12 & University Mission and Vision Statements & low \\
\hline & 13 & Competitive Compensation and Benefits: & low \\
\hline \multirow[t]{5}{*}{ ECONOMIC } & 3 & Geographical location: & low \\
\hline & 14 & Community Support and Service: & low \\
\hline & 15 & Economic Stability and Quality of life: & average \\
\hline & 16 & Demographics/Population of city: & average \\
\hline & 17 & Sustained Employment: & average \\
\hline
\end{tabular}

There were two factors that yielded high significance results: a diverse student population and a multicultural campus environment. The third factor in the 'Diversity' category is faculty diversity, which scored an average level of significance. Table 3 shows the levels of significance for the other polled faculty members. Interestingly, of the factors that were considered, the only one that averaged a noteworthy score was department culture, which ranked low among E\&T faculty. Possible reasons for this event could be the small sample size used or the fact that other black faculty members in different departments are faced with a different set of influential factors that weren't addressed in this study.

Figure 2 breaks down the composite scores in each category for E\&T faculty and other faculty members. The "Diversity' category has the highest composite score for both E\&T and other faculty members though relative comparisons show that the scores differ by more than 20 percentage points. More insight into possible reasons for this result is explored in the next section. For E\&T faculty members the 'Economic' category is the second most significant category, followed by institutional and departmental, respectively. For 'Other' faculty members the decreasing order of significance is departmental, economic and institutional. 
Table 3: Significance of Factors for Other Faculty

\begin{tabular}{|l|l|l|}
\hline Factor & Factor & Level of Significance \\
Number & & \\
\hline 1 & Diverse student population: & low \\
\hline 2 & Faculty diversity: & low \\
\hline 3 & Geographical location: & low \\
\hline 4 & Department culture: & average \\
\hline 5 & Multicultural campus environment: & low \\
\hline 6 & Professional Development Opportunities: & low \\
\hline 7 & Research Opportunities: & low \\
\hline 8 & Course Workload: & low \\
\hline 9 & Size of University: & low \\
\hline 10 & University Reputation: & low \\
\hline 11 & Employment Stability: & low \\
\hline 12 & University Mission and Vision Statements: & low \\
\hline 13 & Competitive Compensation and Benefits: & low \\
\hline 14 & Community Support and Service: & low \\
\hline 15 & Economic Stability and Quality of life: & low \\
\hline 16 & Demographics/Population of city: & low \\
\hline 17 & Sustained Employment: & low \\
\hline
\end{tabular}

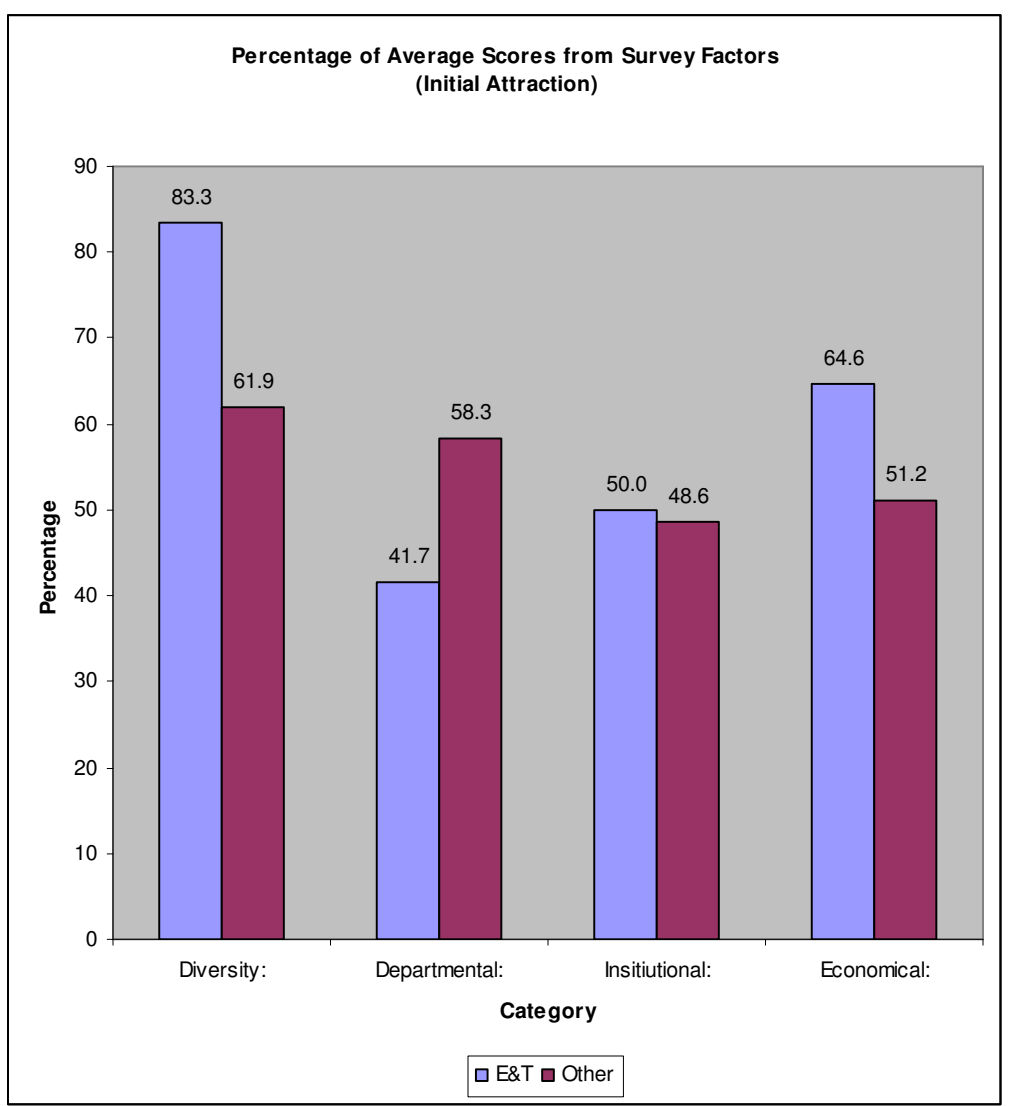

Figure 2-Percentage of Composite Scores for E\&T and Other Groups 
Signal analyses were also conducted to provide more robust performance results. Signal representations of composite scores of each of the factors were created, and they are illustrated in Figure 3.

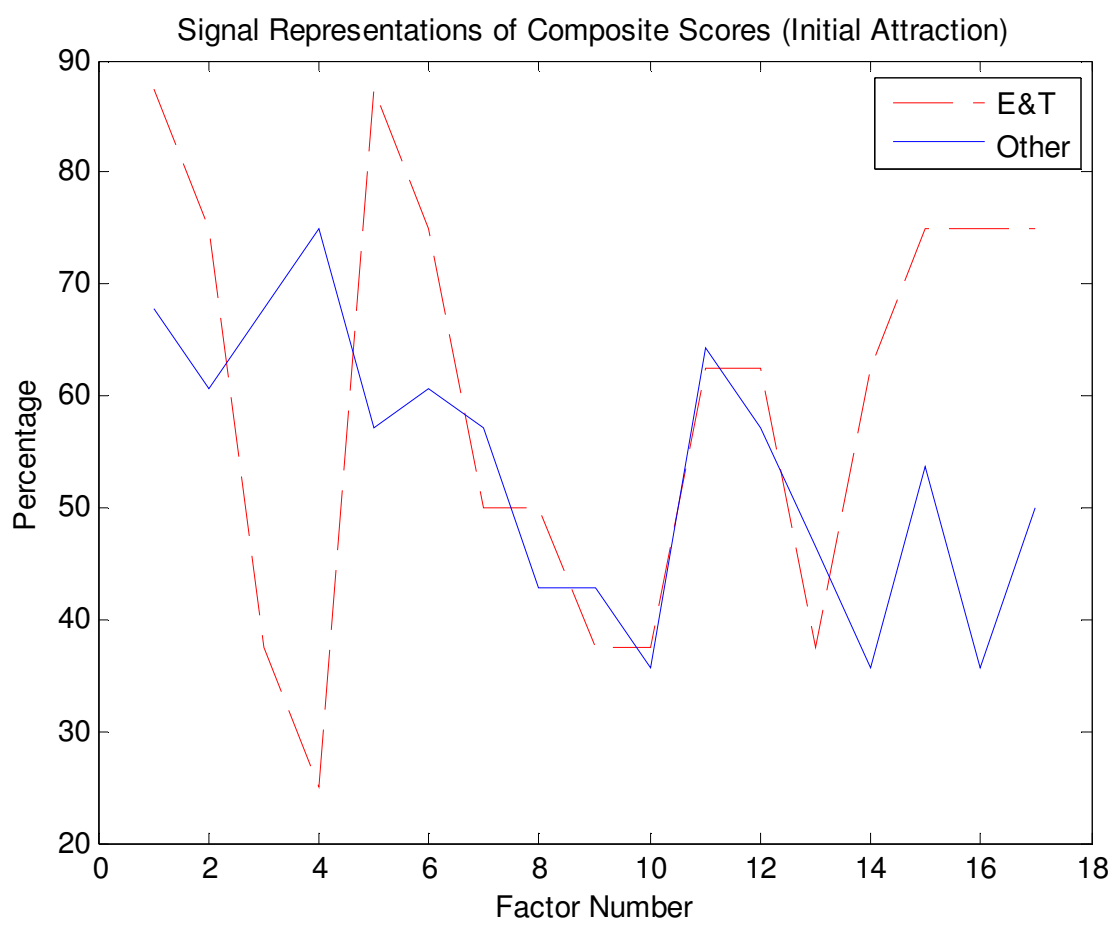

Figure 3-Signal Representations for Composite Scores of Significant Factors

A polynomial fit algorithm was conducted on the 'Other' faculty signal using a least squares assumption. A degree-3 polynomial was chosen in the fit test since the factors were measured on a 4-point agreement scale, which allowed four degrees of freedom. The simulations produced the following polynomial

$$
p(x)=-0.26 x^{3}+2.23 x^{2}-5.85 x+6.70
$$

The E\&T faculty data were evaluated using the polynomial and the prediction error was calculated to determine if the polynomial was adequate to represent the data trends for each factor. The prediction error was determined to be over $50 \%$ for each factor. Consequently, the polynomial was not a good representation of the key trends in the attraction process. From these results, it becomes evident that more empirical data is needed to determine an accurate representation of the factor polynomial for this study.

The normalized significant factor error was also calculated. The results are shown in Figure 4. A factor was negative if the 'Other' group had a higher composite score for that particular factor than the 'E\&T' group. It is clear that for 'Other' black faculty members valued factors 3 and 4 the highest, geographical representation and department culture. For E\&T faculty members, factor 16, demographics of city and factor 5, multicultural campus environment ranked the 
highest. These results indicate that for the E\&T faculty location is an issue as well as multicultural environments.

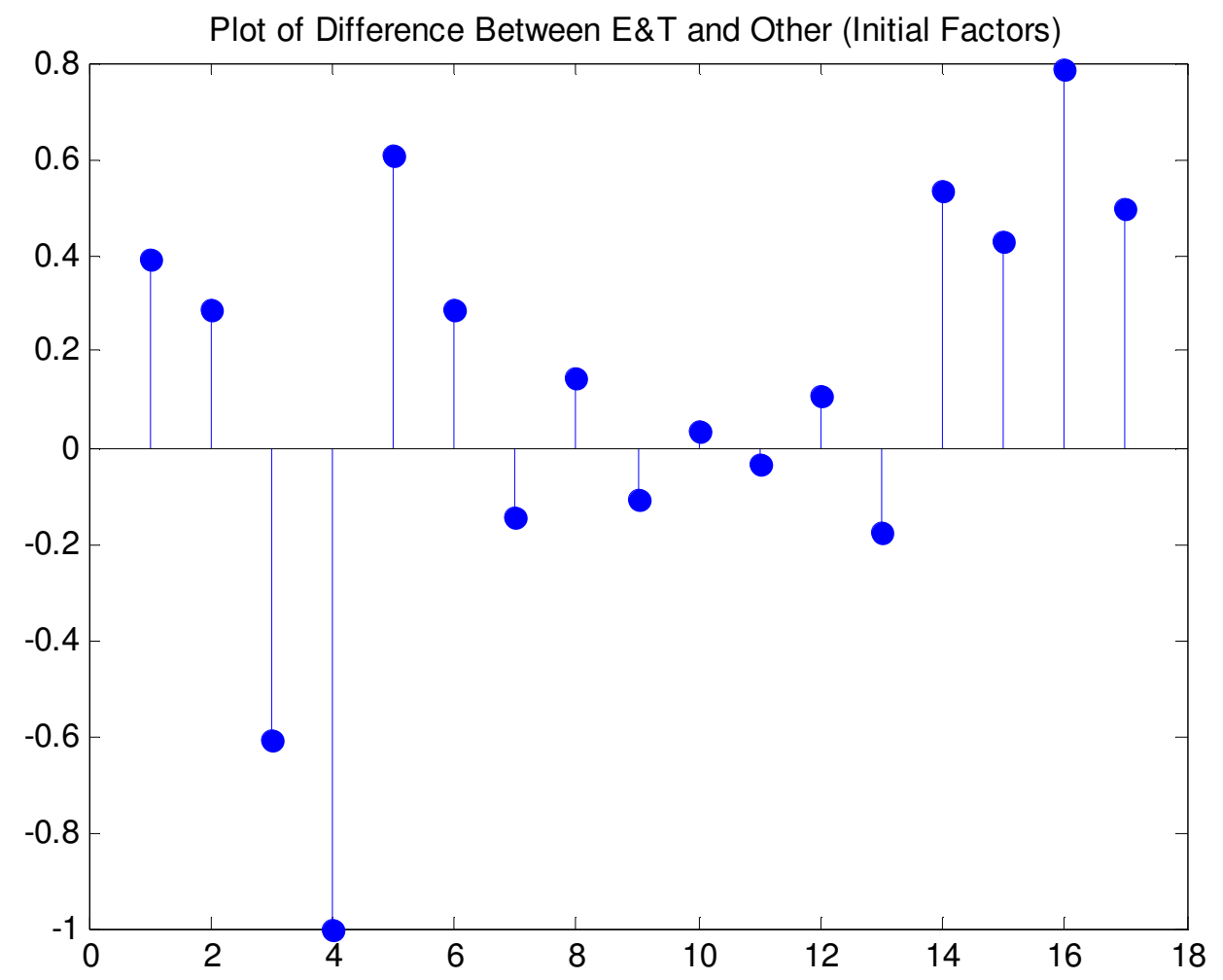

Figure 4-Significant Factor Error between 'E\&T ' and 'Other'

\section{Implications and Future Research}

Several diversity statements were incorporated into the survey to measure attitudes about diversity. Table 4 shows these data results and their corresponding level of significance for E\&T and Other faculty members. The following statements ranked very high among E\&T faculty:

Institutional support and responsibility for the acculturation of minority faculty members should be actively promoted. (Institutional Support)

Diversity of faculty is a significant component of institutional success in regard to attraction, retention, and graduation of a diverse population of quality students. (Diverse Faculty/Student Population)

Affirmative action is one of the key reasons why academic institutions initiate the hiring of minority faculty. (Affirmative Action)

Therefore based on the preliminary work presented in this paper, academic environments that support diverse student populations and multicultural activities are key factors that should be emphasized when attracting qualified minority faculty candidates. Location is another important 
issue, but that factor is not controlled by the department or the institution. However, notable features about the surrounding area may have a positive impact on minority faculty candidates.

Table 4: Level of Significance of Diversity

\begin{tabular}{|l|l|l|}
\hline \multirow{2}{*}{ Diversity Statements } & \multicolumn{2}{|c|}{ Level of Significance } \\
\cline { 2 - 3 } & E\&T & Other \\
\hline Institutional Support & very high & high \\
\hline Departmental Support & average & high \\
\hline Community Support & low & average \\
\hline Role Model Expectations & high & high \\
\hline Unreasonable Service Demands & low & low \\
\hline Lack of Association & low & low \\
\hline Undervalued Research Demands & high & low \\
\hline Undervalued Teaching and Servicehigh & low \\
\hline Diverse Faculty/Student Pop. & very high & high \\
\hline Affirmative Action & very high & average \\
\hline
\end{tabular}

From the work that has been presented, it is clear that higher sample sizes should be obtained for better statistical and comparative results. However based on the results provided, diversity undoubtedly is a key factor in the decision-making process for black engineering and technology faculty members. More data will be collected to provide more analytical and statistical evidence supporting this fact. In addition, attitudes and trends about diversity and black faculty retention is also being explored. There are several interesting data results, particularly in reference to the diversity beliefs of minority Engineering and Technology faculty members (undervalued research demands and undervalued teaching and service demands), that warrant further observations.

\section{Conclusions}

The results indicate that irrespective of the department, diversity is a significant issue in the decision-making process of minority faculty members. Specifically, for Engineering and Technology faculty members diverse student populations and multicultural campus environments were very significant in their decision to join the faculty at this institution. Further analyses are being conducted to collect more data and improve the statistical nature of these results. 


\section{Bibliography}

1. Bernard, Pamela J., "When Seeking a Diverse Faculty, Watch for Legal Minefields", The Chronicle for Higher Education, Diversity in Academic Careers, Volume 53, Number 6, September 29, 2006

2. Elgass, Jane, "Minority Faculty increases despite stiff competition", The University Record, University of Michigan-Ann Arbor, November 23, 1992

3. Woo, Deborah, "The Status of Minority Faculty at UCSC: Recruitment, Retention, and Faculty Diversity Plans", University of California, Santa Cruz, Report on Diversity, August 1995,

4. Mayhew, Matthew J. and Heidi Grunwald, "Factors Contributing to Faculty Incorporation of DiversityRelated Course Content", The Journal of Higher Education, Volume 77, Number 1, 2006

5. Georgia Southern University Faculty Handbook 2006-2007

6. Alberta Learning, "Report of the Working Group on Faculty Attraction and Retention", Business Policy Analysis Adult Learning Division Alberta Learning, 2002 